\title{
Comparative effects of SNX-7081 and SNX-2112 on cell cycle, apoptosis and Hsp90 client proteins in human cancer cells
}

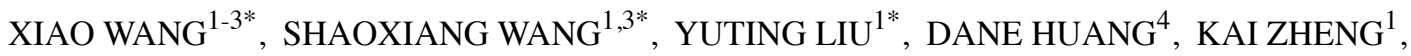 \\ YI ZHANG ${ }^{1}$, XIAOYAN WANG ${ }^{1}$, QIUYING LIU $^{1}$, DEPO YANG ${ }^{2}$ and YIFEI WANG ${ }^{1}$
}

\begin{abstract}
${ }^{1}$ Guangzhou Jinan Biomedicine Research and Development Center, National Engineering Research Center of Genetic Medicine, Jinan University, Guangzhou, Guangdong 510632; ${ }^{2}$ School of Pharmaceutical Sciences, Sun Yat-sen University, Guangzhou, Guangdong 510080; ${ }^{3}$ School of Medicine, Shenzhen University, Shenzhen 518060; ${ }^{4}$ Guangdong Province Engineering Technology Research Institute of Traditional Chinese Medicine, Guangzhou, Guangdong 510095, P.R. China
\end{abstract}

Received June 16, 2014; Accepted August 1, 2014

DOI: $10.3892 /$ or.2014.3552

\begin{abstract}
SNX-2112, a novel 2-aminobenzamide inhibitor of Hsp90, previously showed a broad spectrum of anticancer activity. However, subsequent development has been discontinued due to ocular toxicity as identified in a phase I study. SNX-7081, another closely related Hsp90 inhibitor with a side chain of indole instead of indazole, has recently attracted attention. The aim of the present study was to investigate the anticancer effects of SNX-7081 in eleven cell lines, as well as the mechanisms involved, with SNX-2112 serving as a reference. The cytotoxic effects were determined using an MTT assay and apoptosis was measured using flow cytometry. The results showed that SNX-7081 exerted better inhibitory effects than SNX-2112 in six eighths of the human cancer cell lines, with an average $\mathrm{IC}_{50}$ of $1 \mu \mathrm{M}$. The two inhibitors exerted low cytotoxicity in L-02, HDF and MRC5 normal human cells $\left(\mathrm{IC}_{50}>50 \mu \mathrm{M}\right)$, and arrested cancer cells at the $\mathrm{G} 2 / \mathrm{M}$ phase in a similar manner to normal cells. Compared with SNX-2112, SNX-7081 exhibited more potent effects on cell apoptosis in four sixths of the human cancer cell lines, and was more active in the downregulation of $\mathrm{Hsp} 90$ client proteins. In addition, SNX-7081 exhibited a stronger binding affinity to Hsp90 than
\end{abstract}

Correspondence to: Professor Yifei Wang, Guangzhou Jinan Biomedicine Research and Development Center, National Engineering Research Center of Genetic Medicine, Jinan University, 601 West Huangpu Road, Guangzhou, Guangdong 510632, P.R. China

E-mail: twang-yf@163.com

Professor Depo Yang, School of Pharmaceutical Sciences, Sun Yat-sen University, 132 Waihuan Road, Guangzhou, Guangdong 510080, P.R. China

E-mail: 1ssydp@mail.sysu.edu.cn

*Contributed equally

Key words: Hsp90, SNX-7081, SNX-2112, apoptosis, cell growth, client proteins
SNX-2112 in molecular docking experiments. Considering the superior effects against Hsp90 affinity, cell growth, apoptosis, and Hsp90 client proteins in a majority of human cancer cells, the novel SNX-7081 may be a promising alternative to SNX-2112, which merits further evaluation.

\section{Introduction}

Heat shock protein 90 (Hsp90) is a molecular chaperone with a key role in the preservation of the conformation, stability and function of its client proteins (1). The largest groups of client proteins associated with Hsp90 (e.g., IKK, GSK3, CHK1 and Raf-1) are protein kinases and transcription factors that are important in cellular carcinogenesis (2). Due to the critical roles of Hsp90 client proteins in cancer cell growth arrest, apoptosis and other cancer hallmarks, the inhibition of Hsp90 has received considerable attention as a potential target for cancer treatment (2-4). 17-AAG is the first Hsp90 inhibitor to undergo clinical research, but has several issues related to poor solubility, unacceptable hepatotoxicity and limited bioavailability (5-7). These drawbacks have catalyzed efforts to explore novel scaffolds with improved pharmacological properties for clinical applications.

SNX-2112, a novel Hsp90 inhibitor with the 2-aminobenzamide scaffold, has been widely used in cancer research (8-12). SNX-2112 exerts its growth inhibitory and apoptosis-inducing activity on many human cancer cells $(1,8,11,13)$. However, subsequent development has been discontinued due to ocular toxicity observed in animal models and in a separate phase I study (14). Developing alternatives of SNX-2112 based on the 2-aminobenzamide scaffold may be a feasible method for meeting clinical needs. SNX-7081 is another Hsp90 inhibitor derived from 2-aminobenzamide and optimized by SAR explorations (15), with a side-chain of indole instead of indazole at SNX-2112. To the best of our knowledge, SNX-7081 has not attracted enough attention in cancer therapy, and is only limited to investigations on its potential in chronic lymphocytic leukemia and inflammatory diseases (16-19).

In the present study, we tested the anticancer effects and examined the mechanisms of SNX-7081 using several human cancer cells in vitro, with SNX-2112 as a reference. 
Table I. IC $_{50}$ values of SNX-7081 and SNX-2112.

\begin{tabular}{lcc}
\hline & \multicolumn{2}{c}{ Drug: $\mathrm{IC}_{50}(\mu \mathrm{M})$} \\
\cline { 2 - 3 } Cell lines & SNX-7081 & SNX-2112 \\
\hline Human cancer cells & & \\
K562 (leukemia) & $<0.01$ & $<0.01$ \\
A375 (melanoma) & $0.26 \pm 0.12^{\mathrm{a}}$ & $0.55 \pm 0.22$ \\
MCF-7 (breast cancer) & $1.09 \pm 0.74$ & $2.58 \pm 1.30$ \\
Hep-2 (laryngeal cancer) & $1.13 \pm 0.64^{\mathrm{a}}$ & $3.58 \pm 0.95$ \\
HepG2 (liver cancer) & $1.29 \pm 0.51$ & $2.52 \pm 1.35$ \\
A549 (lung cancer) & $3.61 \pm 1.50^{\mathrm{a}}$ & $5.86 \pm 2.24$ \\
SW-620 (colon carcinoma) & $0.05 \pm 0.03$ & $<0.01$ \\
HeLa (cervical cancer) & $9.16 \pm 4.50$ & $6.31 \pm 3.34$ \\
Normal human cells & & \\
L-02 (liver cells) & $>100.00$ & $>100.00$ \\
HDF (dermal fibroblasts) & $>100.00$ & $>100.00$ \\
MRC5 (fetal lung fibroblasts) & $70.16 \pm 27.85$ & $>100.00$ \\
\hline
\end{tabular}

Cells were cultured with various concentrations $(0-10 \mu \mathrm{M}$ in cancer cells, 0-50 $\mu \mathrm{M}$ in normal cells) of compounds for $72 \mathrm{~h}$. $\mathrm{IC}_{50}$ values of SNX-7081 and SNX-2112 towards eight cancer cell lines (K562, A375, MCF-7, Hep-2, HepG2, A549, SW620 and HeLa) and three human normal cells (L-02, MRC5 and HDF) at $72 \mathrm{~h}$ as determined by using MTT assay. Data are the means \pm SD of three replicates $\left({ }^{\mathrm{a}} \mathrm{P}<0.05\right)$.

To demonstrate more details involved in the antitumor effects of the two compounds, we investigated Hsp90 affinity, cell growth, cell cycle, apoptosis and the expression of Hsp90 clients in various cancer cells.

\section{Materials and methods}

Reagents and antibodies and cells. SNX-7081 and SNX-2112 were prepared in our laboratory with purities of $>98.0 \%$ according to a previously described procedure $(20,21)$, dissolved in $10 \mathrm{mM}$ dimethyl sulfoxide (DMSO) as a stock solution and stored at $-20^{\circ} \mathrm{C}$. Antibodies against IKK $\alpha$, Raf-1, CHK1 and GSK3 $\beta$ were purchased from Epitomics (Burlingame, CA, USA), and the glyceraldehyde-3-phosphate dehydrogenase (GAPDH) antibody was obtained from Millipore (Billerica, MA, USA).

Cell culture. Eleven cell lines, including eight human cancer cell lines and three human normal cell lines, were used in the present study (Table I). Primary HDF cells were isolated from the foreskins of newborns using a previously reported procedure $(22,23)$. All other cell lines were purchased from the Cell Bank of the China Science Academy (Shanghai, China). CML K562 cells, A375 melanoma cells, the normal human diploid fibroblast (HDF) and the normal human liver L-02 cells were cultured in RPMI-1640 medium. MCF-7 breast cancer cells, Hep-2 laryngeal epidermoid carcinoma, HepG2 liver carcinoma, A549 lung adenocarcinoma, HeLa cervical carcinoma and SW-620 colon carcinoma cells were cultured in Dulbecco's modified Eagle's medium (DMEM). The normal human fibro- blastic cell line (MRC-5) was cultured in Minimum Essential Medium (MEM). Cells were maintained at $37^{\circ} \mathrm{C}$ in a $5 \%$ $\mathrm{CO}_{2}$ atmosphere. All the media were supplemented with $10 \%$ heat-inactivated fetal bovine serum (FBS) supplemented with $50 \mathrm{U} / \mathrm{ml}$ penicillin and streptomycin.

MTT assay. Exponentially growing cells were seeded in 96-well culture plates $\left(5-10 \times 10^{3}\right.$ cells/well) and allowed to adhere overnight. Cancer cells were incubated with SNX-7081 or SNX-2112 at various concentrations $(0-10 \mu \mathrm{M})$ for $72 \mathrm{~h}$. HDF, MRC5 and L-02 normal human cells were incubated with drugs at various concentrations $(0-50 \mu \mathrm{M})$ for $72 \mathrm{~h}$, along with an equal volume of DMSO as the solvent control. MTT solution $(10 \mu \mathrm{l})$ was added to each well $(0.5 \mathrm{mg} / \mathrm{ml})$ for an additional 4-h incubation $\left(37^{\circ} \mathrm{C}, 5 \% \mathrm{CO}_{2}\right)$. The precipitated formazan was dissolved in $100 \mu \mathrm{l}$ of DMSO. A 96-well multiscanner autoreader (M450; Bio-Rad Laboratories, Hercules, CA, USA) was used to measure the absorbance of each well at $570 \mathrm{~nm}$, with a reference wavelength of $630 \mathrm{~nm}$. The $\mathrm{IC}_{50}$ values, defined as the drug concentration that caused 50\% inhibition of absorbance compared with the control cells treated with DMSO only, were calculated using the PrismPad program.

Cell cycle analysis. Cell cycle distribution was determined by DNA staining with propidium iodide (PI) (13). Briefly, cancer cell lines were cultured and treated in 6-well culture plates $\left(6 \times 10^{5}\right.$ cells/well) with SNX-7081 or SNX-2112 $(1.0 \mu \mathrm{M})$ for $48 \mathrm{~h}$. The cells were then washed in phosphate-buffered saline (PBS) and fixed in $70 \%$ ethanol overnight. The cells were collected and resuspended in PBS containing $50 \mu \mathrm{g} / \mathrm{ml}$ PI, $0.1 \mathrm{mg} / \mathrm{ml}$ RNase, and $5 \%$ Triton X-100 and incubated at $37^{\circ} \mathrm{C}$ for $30 \mathrm{~min}$. Subsequently, the cells were analyzed on a flow cytometer (Becton-Dickinson, San Jose, CA, USA), and the percentages of cells present in different phases of the cell cycle were analyzed using the CellQuest software (Becton-Dickinson).

Apoptosis assay. Apoptosis was measured by flow cytometry after staining with Annexin V-FITC and PI according to the instructions of the Annexin V-FITC/PI staining kit (Nanjing KeyGen Biotech., Co., Ltd., Nanjing, China). Briefly, cancer cells were cultured in the presence of the indicated concentrations of SNX-7081 or SNX-2112 $(1.0 \mu \mathrm{M})$ for $48 \mathrm{~h}$, harvested, washed twice and resuspended in $500 \mu 1$ of $1 \mathrm{X}$ binding buffer containing Annexin V-FITC and PI. Samples were incubated at room temperature for $10 \mathrm{~min}$ and analyzed by FACS.

Immunoblotting. K562 cells were incubated with $1.0 \mu \mathrm{M}$ SNX-7081 or SNX-2112 for 0, 6, 12, 24 or $48 \mathrm{~h}$. Whole-cell lysates were prepared by washing the cells with PBS and subjecting them to lysis with RIPA buffer for $30 \mathrm{~min}$ on ice. Total protein concentrations of whole-cell lysates were determined using the BCA protein assay kit. Equal amounts of protein samples were loaded onto 8-12\% sodium dodecyl sulfate (SDS) polyacrylamide gel electrophoresis (PAGE) gels . After electrophoresis, the proteins were transferred to polyvinylidene fluoride (PVDF) membranes (Millipore), examined with primary antibodies and then incubated with horseradish peroxidase (HRP)-conjugated secondary antibodies. Specific protein bands were visualized using the chemiluminescence method and imaged by autoradiography. Any differences in 

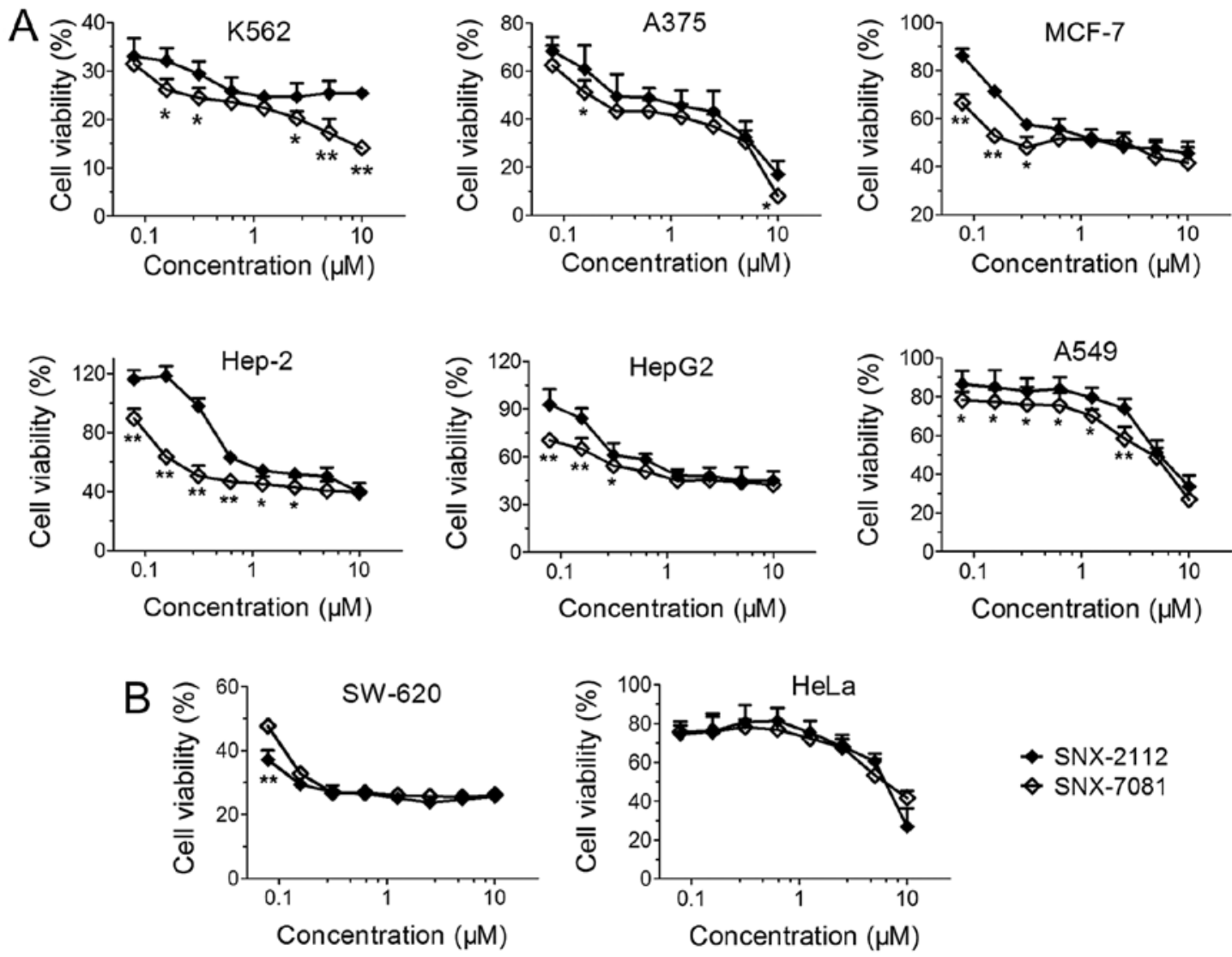

Figure 1. Cytotoxic effects of SNX-7081 and SNX-2112 on human cancer cell lines were determined by the MTT assay. (A) K562, A375, MCF-7, Hep-2, HepG2, A549 and (B) SW-620 and HeLa cells were treated with various concentrations of SNX-7081 and SNX-2112 for $72 \mathrm{~h}$. Percentages of viable cells were calculated by comparing treated and solvent control cells. Data are the means $\pm \mathrm{SD}$ of three replicates $\left({ }^{*} \mathrm{P}<0.05 ;{ }^{* *} \mathrm{P}<0.01\right)$.

protein loading were normalized to the corresponding levels of the GAPDH control.

Docking assay. The affinity of SNX-7081 and SNX-2112 against Hsp90 was determined by the MOE docking assay. The crystal structure of Hsp90 was taken from the Protein Data Bank (PDB code: 3R92). The two compounds were converted to 3D structures, and energy was minimized in MOE. The binding site of Hsp90 was minimized using the AMBER 99 force field in MOE with the default parameter. The two compounds were docked, employing Triangle Matcher as the placement method and the function London $\mathrm{dG}$ as the first scoring function. The refinement was set to force field (AMBER 99), and the docked poses were energy minimized in the receptor pocket (24). The conformations of lowest energy were given, and a lower scoring value indicated a more favorable binding.

Statistical analysis. Data were evaluated by the Welch t-test when only two value sets were compared. One-way ANOVA followed by the Dunnett's test was used for more group comparisons if $\geq 3$ experiments were involved. Results were expressed as means \pm SD with a significance at ${ }^{*} \mathrm{P}<0.05$ or ${ }^{* *} \mathrm{P}<0.01$.

\section{Results}

Anti-proliferative effects of SNX-7081 and SNX-2112 on hunman cancer cells. The growth inhibitory effects of SNX-7081 and SNX-2112 on human cancer lines originating from bone marrow, colon, skin, larynx, breast, liver, cervix and lung were comopared. Cells were exposed to various concentrations $(0-10 \mu \mathrm{M})$ for $72 \mathrm{~h}$ and cell viability was quantified by the MTT assay. SNX-7081 and SNX-2112 significantly inhibited the growth of eight human cancer cells in a dose-dependent manner. The cell viability of SNX-7081 was significantly lower than that of SNX-2112 at the low concentration $(<1 \mu \mathrm{M})$ in K562, A375, MCF-7, Hep-2, HepG2 and A549 cells (Fig. 1A). In addition, SNX-7081 exhibited similar or weaker effects to SNX-2112 in SW-620 and HeLa cells (Fig. 1B). The $\mathrm{IC}_{50}$ values of SNX-7081 and SNX-2112 for these cells were always $\sim 1 \mu \mathrm{M}$ (Table I). For the remaining experiments, $1 \mu \mathrm{M}$ was applied as the optimal concentration.

Cytotoxic effects of SNX-7081 and SNX-2112 on human normal cells. The cytotoxic effects of two agents on three human normal cell lines (L-02, HDF and MRC5 cells) were determined by the MTT assay. The cells were exposed to SNX-7081 or SNX-2112 at concentrations of 3.13-50.0 $\mu \mathrm{M}$ for $72 \mathrm{~h}$. The $\mathrm{IC}_{50}$ value could not be determined even at the high concentration of $50.0 \mu \mathrm{M}$ (Table I and Fig. 2), suggesting that SNX-7081 cytotoxicity was lower as compared to SNX-2112 in L-02, MRC5 and HDF cells. Therefore, SNX-7081 and SNX-2112 were provided with acceptable cytotoxicity toward normal cells and high selectivity in cancer cells.

Induction of cell cycle arrest at G2/M phase by SNX-7081 and $S N X-2112$. To explore the anti-proliferative mechanism 

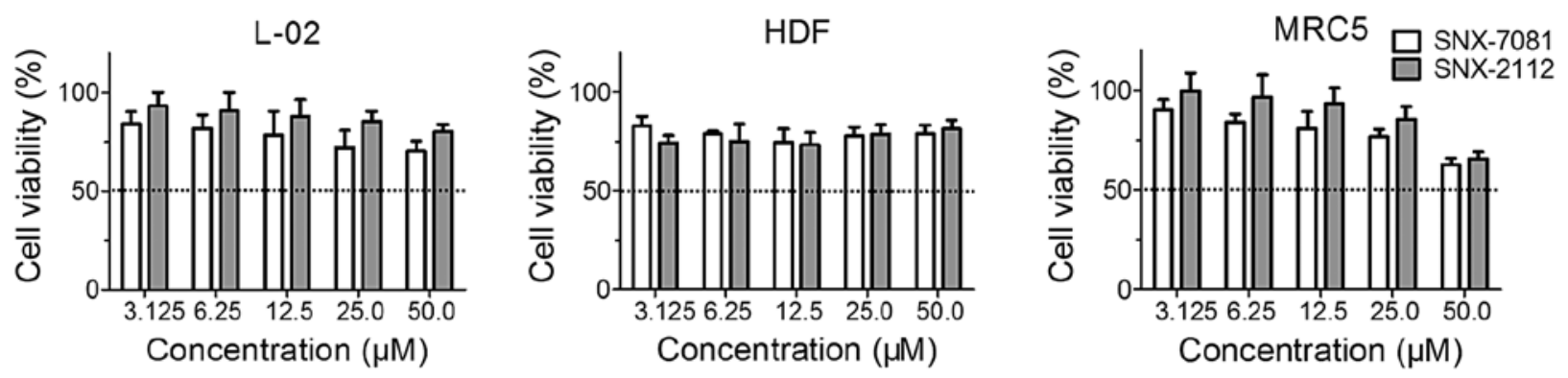

Figure 2. Cytotoxic effects of SNX-7081 and SNX-2112 on normal human cell lines were determined by the MTT assay. Cells were treated with various concentrations of SNX-7081 and SNX-2112 for $72 \mathrm{~h}$. Percentages of viable cells were calculated by comparing treated and solvent control cells. Data are the means $\pm \mathrm{SD}$ of three replicates.
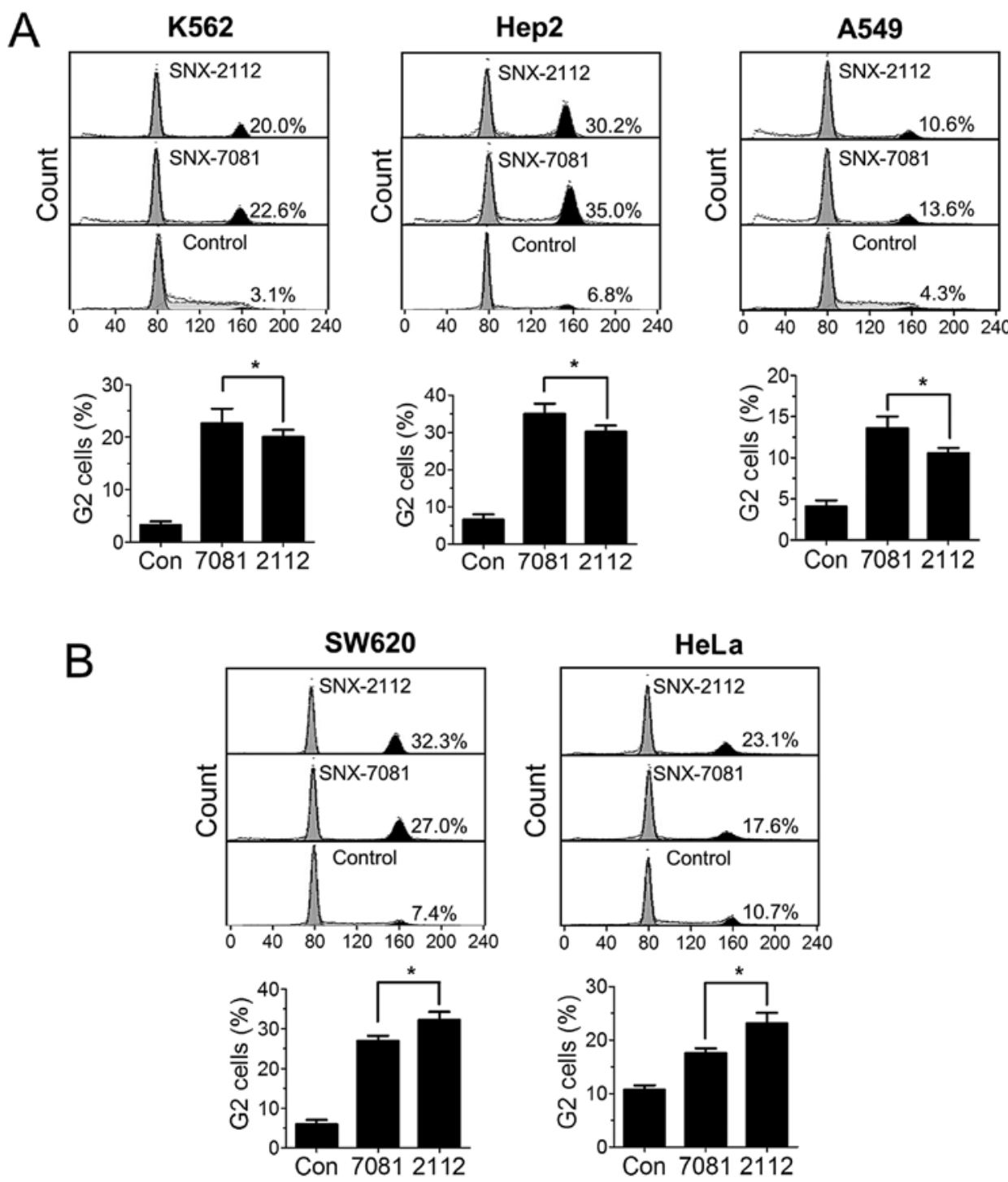

Figure 3. Cell cycle analysis of cancer cells after treatment with SNX-7081 and SNX-2112 in five cancer cells lines. (A) K562, Hep-2, A549 and (B) SW-620 and HeLa cells were cultured with SNX-7081 and SNX-2112 at $1 \mu \mathrm{M}$, collected after $48 \mathrm{~h}$ and stained with PI. The DNA content and cell cycle distribution were analyzed by flow cytometry. Results are the means \pm SD of three replicates. Representative images from three experiments are shown, and percentages of cells in cycle arrest are indicated. Data are the means $\pm \mathrm{SD}$ of three replicates $(\mathrm{P}<0.05)$.

of SNX-7081 and SNX-2112, we assessed their effects on the cell cycle distribution in the K562, Hep-2, A549, SW620 and HeLa cancer cell lines using flow cytometry. Cells treated with SNX-7081 and SNX-2112 $(1 \mu \mathrm{M})$ for $48 \mathrm{~h}$, were subjected to flow cytometric analysis after PI staining. SNX-7081 more effectively increase the cell amount of G2/M phase in K562, Hep-2 and A549 cells (Fig. 3A), while SNX-2112 induces a more significant G2/M arrest in SW-620 and HeLa cells 


\section{A

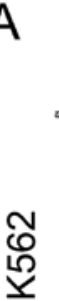
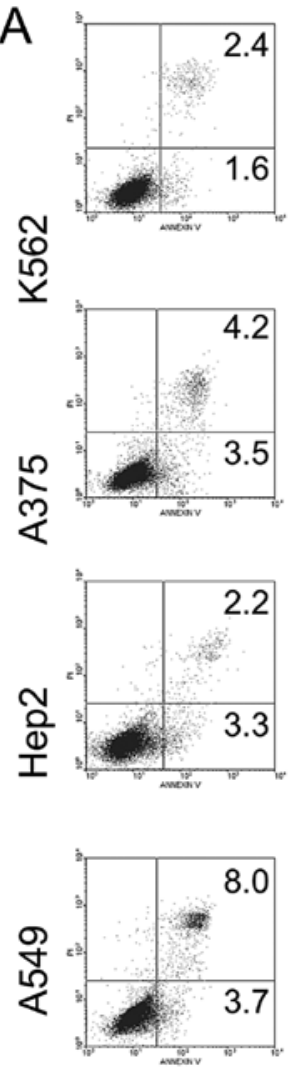

Control

B
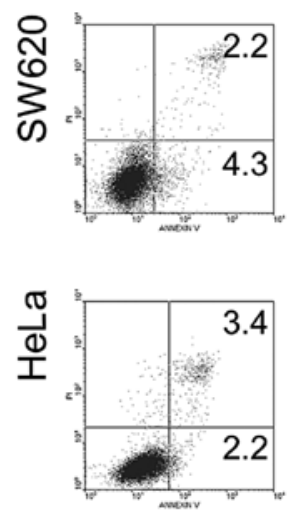

Control
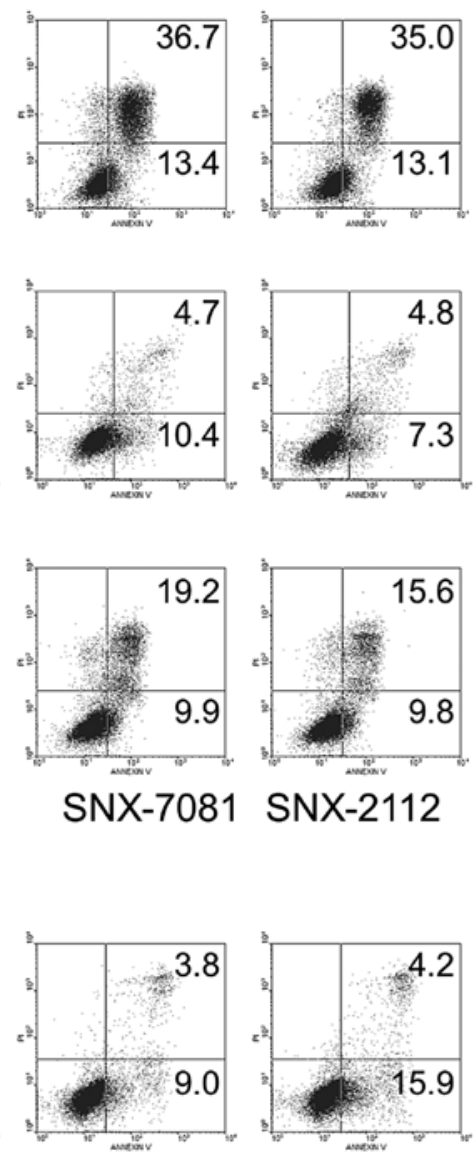

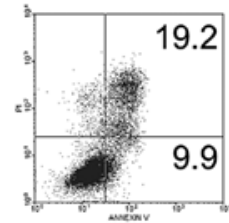

SNX-7081

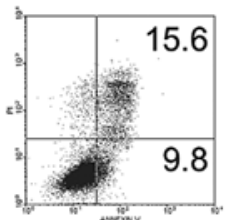

SNX-2112
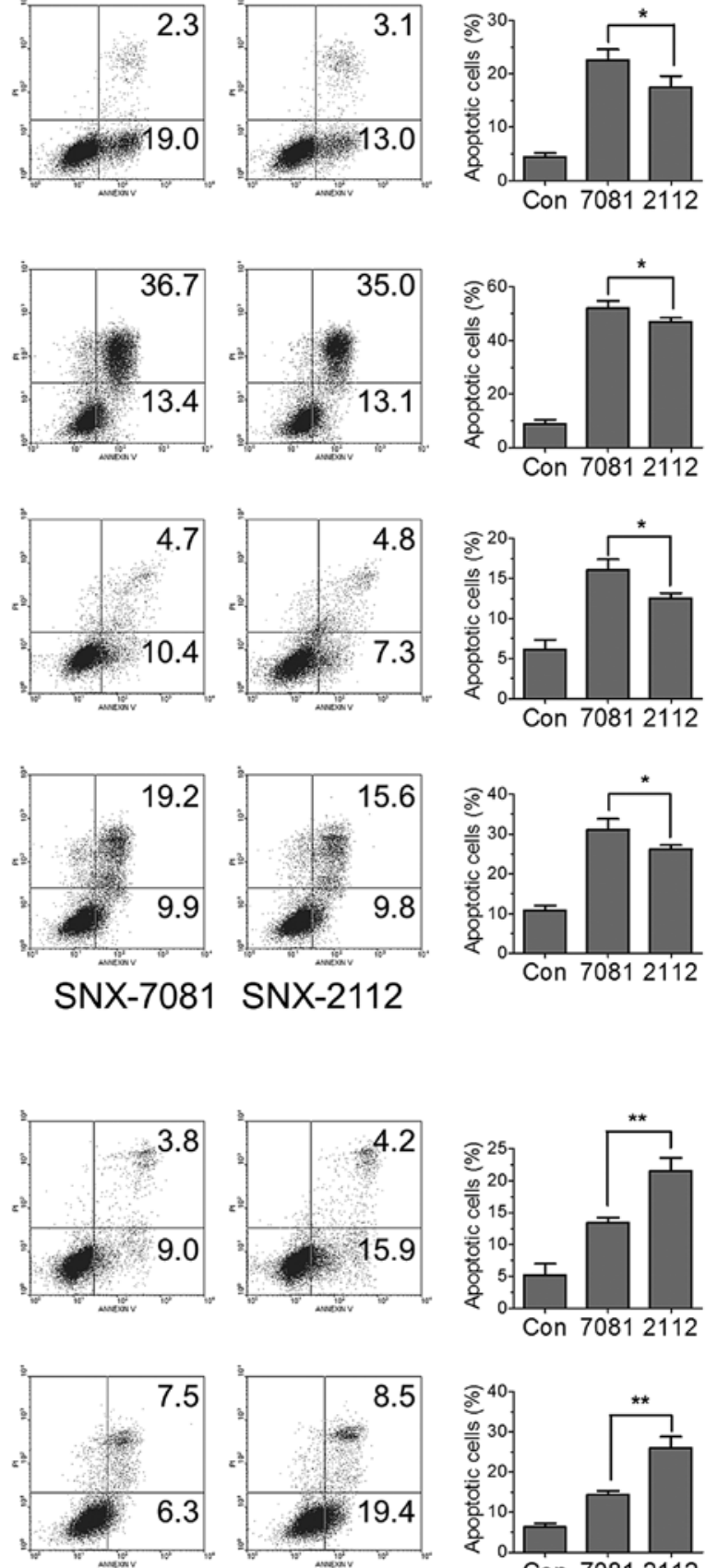

SNX-7081

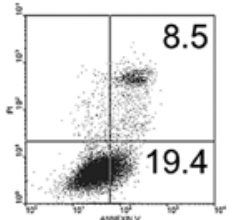

SNX-2112

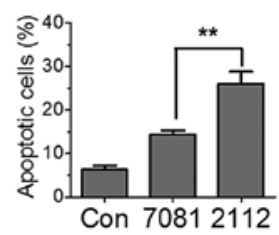

Figure 4. Effects of SNX-7081 and SNX-2112 on apoptosis induction in human cancer cells. (A) K562, A375, Hep-2, A549 and (B) SW-620 and HeLa cells were treated with control (vehicle), SNX-7081 and SNX-2112 at $1 \mu \mathrm{M}$ for $48 \mathrm{~h}$. Apoptosis was detected by flow cytometric analysis based on PI (y-axis) and Annexin V (x-axis) staining. Representative images from three experiments are shown, and the percentages of apoptotic cells (lower and upper rightquadrants) are indicated. Data are the means $\pm \mathrm{SD}$ of three replicates $\left({ }^{*} \mathrm{P}<0.05 ;{ }^{* *} \mathrm{P}<0.01\right)$.

(Fig. 3B). The finding suggested that SNX-7081-induced inhibitory effects may be mediated by cell cycle arrest.

Effects of SNX-7081 and SNX-2112 on cancer cell apoptosis. To examine whether apoptosis is involved in the inhibition of cancer cell proliferation by SNX-2112 or SNX-7081, cancer cells were treated with $1 \mu \mathrm{M} \mathrm{SNX}-2112$ or SNX-7081 for $48 \mathrm{~h}$ and subjected to Annexin V- and PI-double staining for flow cytometric analysis. The degree of apoptosis was calculated as the sum of the percentages of cells in the lower right and upper right quadrants. SNX-7081 and SNX-2112 clearly induced apoptosis in all six cell lines. SNX-7081 induced more apoptosis in K562, Hep-2 and A549 cells (Fig. 4A), but less apoptosis in SW-620 and HeLa cells as compared to that of SNX-2112 (Fig. 4B). The data indicated that SNX-7801induced apoptosis in some cancer cells was more potent than SNX-2112.

Effects of SNX-7081 and SNX-2112 on the expression of Hsp90 client proteins. The basic mechanism of Hsp90 inhibitors involves binding to the ATP pocket and suppressing the ATPase activity of Hsp90, resulting in the degradation of 

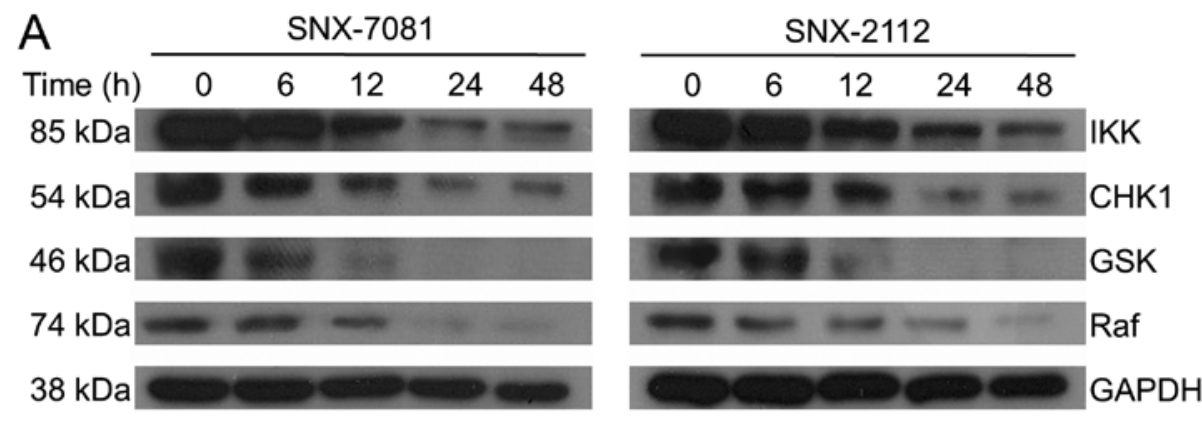

B
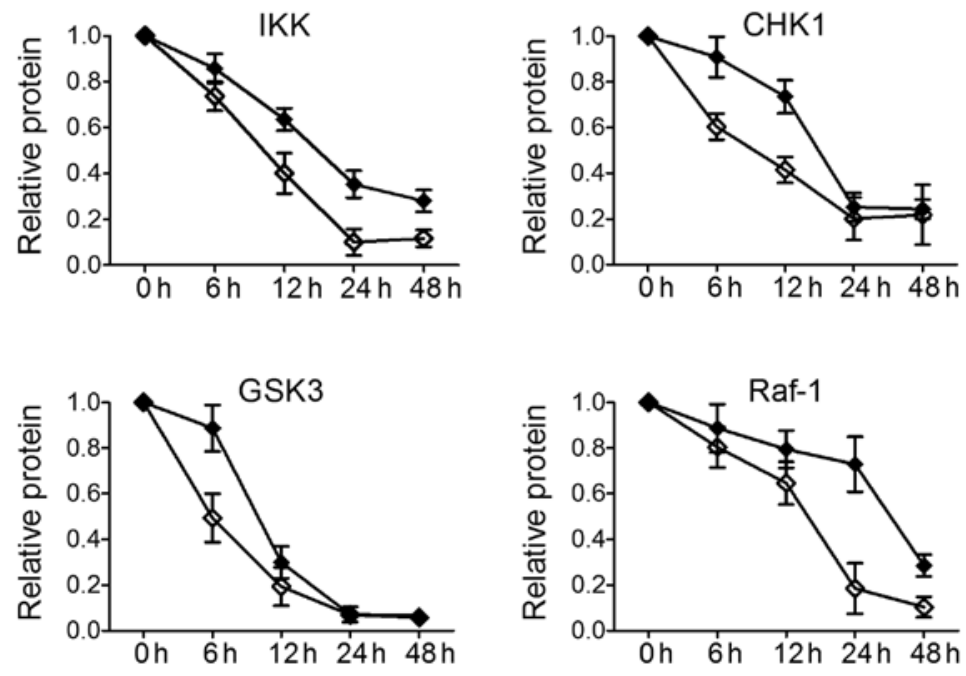

Figure 5. Inhibition of Hsp90 client proteins by SNX-7081 and SNX-2112. (A) K562 cells were cultured with control (vehicle), SNX-2112 and SNX-7081 at $1 \mu \mathrm{M}$ for the indicated times. Equal amounts of whole-cell lysates $(25-50 \mu \mathrm{g})$ were analyzed by western blot analysis to detect the expression of Hsp90 client proteins. (B) In the accompanying bar graphs, protein levels were normalized to GAPDH, which was used as a loading control. Results are shown as means \pm SD of three independent experiments.

its client proteins (25). It was previously demonstrated that SNX-2112 led to downregulation of the Hsp90 client proteins Bcr-abl and Akt in K562 cells (13). In the present study, we compared the effect of SNX-7081 and SNX-2112 on the expression of other essential Hsp90 client proteins, including IKK $\alpha$, GSK3, CHK1 and Raf-1, which are crucial for the growth of cancer cells. Western blot analysis showed that the levels of these proteins were reduced in a time-dependent manner following exposure to the compounds (Fig. 5). After treatment with $1 \mu \mathrm{M}$ of SNX-7081 for $48 \mathrm{~h}$, the expression of IKK $\alpha$, CHK1, GSK3 and Raf-1 was significantly decreased to 11.7 , $21.8,5.8$ and $10.4 \%$, respectively. SNX-7081 exerted stronger inhibitory effects on the expression levels of Hsp90 client proteins than SNX-7081. These data support the principle that SNX-7081 and SNX-2112 act via the inhibition of Hsp90 chaperone function, and the SNX-7081 was more potent in suppressing Hsp90 client proteins.

Binding affinity of SNX-7081 and SNX-2112 to Hsp90. To determine the binding affinity of SNX-7081 and SNX-2112 to Hsp90, the inhibitors were docked into the ATP site of Hsp90, using MOE. A lower scoring value suggests a more favorable binding. The ligand-receptor interaction maps for SNX-7081 and SNX-2112 are shown in Fig. 6. We found two hydrogen bond residues (Phe-138 and Trp-163), two side chain donor molecules (Lys-58 and Thr-184), and one side chain acceptor molecule (Asp-93) that bound to SNX-7081. SNX-7081 interacted with five residues in the pocket, while SNX-2112 only interacted with three residues. The scoring value of SNX-7081 was $-33.53 \mathrm{kcal} / \mathrm{mol}$, which was lower than the $-30.94 \mathrm{kcal} /$ mol on SNX-2112. It was evident that SNX-7081 was the highly-scored compound, suggesting that SNX-7081 had a stronger binding affinity to Hsp90 than SNX-2112.

\section{Discussion}

The discontinuity in clinical trials of SNX-2112 results in opportunities and challenges for other 2-aminobenzamide inhibitors of Hsp90. In the present study, we found that SNX-7081, an analogue of SNX-2112, exhibited higher affinity for Hsp90 than SNX-2112 in molecular docking experiments. The structure of SNX-7081 is different from SNX-2112, with a side chain of indole instead of indazole. In addition, the methyl substituent in the indole ring instead the of 5-fluoro substituent in indazole may also contribute to the higher affinity of SNX-7081. A similar binding model suggested that SNX-7081 may be a feasible alternative of SNX-2112, leading to a comparison of the anticancer activity of the two compounds. 

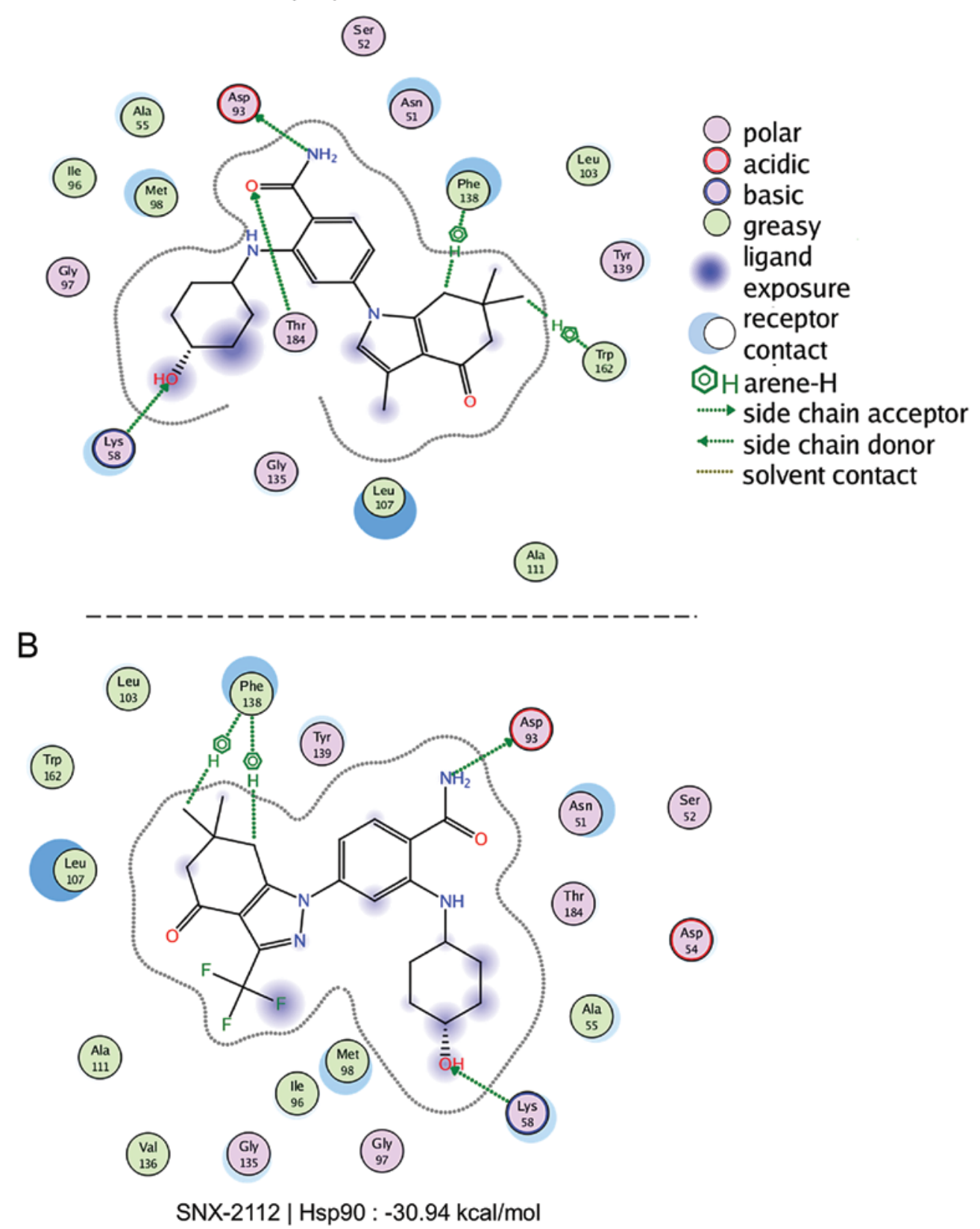

Figure 6. Molecular modeling of SNX-7081 and SNX-2112 binding with Hsp90. Docking of (A) SNX-7081 and (B) SNX-2112 into the N-terminal domain of Hsp90 (protein structure with PDB code 3R92). The conformations of lowest energy are shown, and a lower scoring value indicates a more favorable binding.

SNX-7081 had better activity than SNX-2112 in most cancer cells. One interpretation of this finding is that the expressions and functions of various Hsp90 isoforms are not uniform in different cell types. These Hsp90 isoforms include Hsp90 $\alpha$ and Hsp90 $\beta$ in the cytoplasm and nucleus, GRP94 in the endoplasmic reticulum (ER) and TRAP1 in the mitochondria (25). The functions of Hsp90 $\alpha$ include stress-induced cytoprotection and cell cycle regulation, whereas Hsp90 $\beta$ plays an important role in early embryonic development, signal transduction and long-term cell adaptation $(26,27)$. GRP94 is specialized for protein folding and plays a role in the degradation of misfolded proteins through the ER-associated degradation pathway (28). Additionally, TRAP1 is involved in signal transduction, protein folding, protein degradation and morphologic evolution (29). Thus, the development of Hsp90 inhibitors against specific Hsp90 isoforms is a promising strategy for cancer therapy.

In addition, SNX-7081 and SNX-2112 exhibited strong selectivity in cell growth inhibition between cancer cells and normal cells. This is probably because the expression and molecular conformation of Hsp90 are greatly different in cancer and normal cells. In tumor cells, Hsp90 may be exclusively complexed with co-chaperones, forming a superchaperone complex in a state of high affinity for ATP/ADP or ligands (e.g., Hsp90 inhibitors) of this regulatory pocket. However, in normal cells, Hsp90 is predominantly in a 
latent, uncomplexed and low affinity state $(30,31)$. Therefore, compared with Hsp90 in normal cells, tumor Hsp90 is hypersensitive to Hsp90 inhibitors.

Results of this study show that SNX-7081 exhibits an antitumor profile of the natural product Hsp90 inhibitors: growth inhibition, G2 cell cycle arrest, cell apoptosis induction and degradation of Hsp90 clients (32). Previously, it was found that SNX-2112 can induce the degradation of Bcr-abl and Akt in K562 cells (21). Although there are many other Hsp90 client proteins, the focus of the present study was on the levels of IKK $\alpha$, GSK3, CHK1 and Raf-1, due to their critical roles in the proliferation of human leukemia cells. For instance, IKK $\alpha$ is an NF- $\kappa \mathrm{B}$ inhibitory protein that contributes to the de-regulated expression of various cellular genes in T-cell leukemia cells $(33,34)$. GSK 3 controls cell survival, and its inactivation induces cell apoptosis in leukemia cells $(35,36)$. Inhibition of CHK1 kinase has been shown to induce cell cycle arrest in K562 cells (37), while targeting Raf- 1 gene expression by a DNA enzyme inhibits the growth of leukemia cells (38). Therefore, the downregulation of Hsp90 client proteins in the upstream pathway may be the mechanism of the induction of growth inhibition, cell cycle arrest and apoptosis pertaining to SNX-7081 in cancer cells.

In conclusion, we have demonstrated that SNX-7081 was significantly more potent than SNX-2112 in a majority of human cancer cells. Consequently, this superiority effect merits further confirmation in xenograft experiments in vivo. The mode of action of antitumor activity may be associated with the induction of cell cycle arrest, apoptosis and Hsp90 client proteins degradation. Our results suggest that the novel Hsp90 inhibitor SNX-7081 is a promising alternative to SNX-2112, although more fundamental investigations are required to confirm the results.

\section{Acknowledgements}

We would like to thank Professor Bao-Jian Wu from Jinan University (Guangzhou, China) for proofreading the manuscript. The present study was supported by grants from the National Natural Science Foundation of China (grant no. 81201727), the China Postdoctoral Science Foundation (grant nos. 2012M511882 and 2013T60827), the open project of State Key Laboratory of Molecular Oncology (SKL-KF2013-14), the Guangdong Province and Ministry of Education Ministry of Science and Technology Products Research Combined Platform Project (grant no. 2010B091000013), and the Natural Science Foundation of Guangdong Province (grant no. S2012040006873).

\section{References}

1. Taipale M, Jarosz DF and Lindquist S: HSP90 at the hub of protein homeostasis: emerging mechanistic insights. Nat Rev Mol Cell Biol 11: 515-528, 2010.

2. Trepel J, Mollapour M, Giaccone G and Neckers L: Targeting the dynamic HSP90 complex in cancer. Nat Rev Cancer 10: 537-549, 2010.

3. Hanahan D and Weinberg RA: Hallmarks of cancer: the next generation. Cell 144: 646-674, 2011

4. Mahalingam D, Swords R, Carew JS, Nawrocki ST, Bhalla K and Giles FJ: Targeting HSP90 for cancer therapy. Br J Cancer 100 1523-1529, 2009.
5. Banerji U, O'Donnell A, Scurr M, et al: Phase I pharmacokinetic and pharmacodynamic study of 17-allylamino, 17-demethoxygeldanamycin in patients with advanced malignancies. J Clin Oncol 23: 4152-4161, 2005.

6. Taldone T, Gozman A, Maharaj R and Chiosis G: Targeting Hsp90: small-molecule inhibitors and their clinical development. Curr Opin Pharmacol 8: 370-374, 2008.

7. Goetz MP, Toft D, Reid J, et al: Phase I trial of 17-allylamino17-demethoxygeldanamycin in patients with advanced cancer. J Clin Oncol 23: 1078-1087, 2005.

8. Chinn DC, Holland WS, Yoon JM, Zwerdling T and Mack PC: Anti-tumor activity of the HSP90 inhibitor SNX-2112 in pediatric cancer cell lines. Pediatr Blood Cancer 58: 885-890, 2012.

9. Wang R, Shao F, Liu Z, et al: The Hsp90 inhibitor SNX-2112, induces apoptosis in multidrug resistant K562/ADR cells through suppression of Akt/NF-kappaB and disruption of mitochondriadependent pathways. Chem Biol Interact 205: 1-10, 2013.

10. Wang SX, Ju HQ, Liu KS, et al: SNX-2112, a novel Hsp90 inhibitor, induces G2/M cell cycle arrest and apoptosis in MCF-7 cells. Biosci Biotechnol Biochem 75: 1540-1545, 2011.

11. Bachleitner-Hofmann T, Sun MY, Chen CT, et al: Antitumor activity of SNX-2112, a synthetic heat shock protein-90 inhibitor, in MET-amplified tumor cells with or without resistance to selective MET inhibition. Clin Cancer Res 17: 122-133, 2011.

12. Liu KS, Liu H, Qi JH, et al: SNX-2112, an Hsp90 inhibitor, induces apoptosis and autophagy via degradation of Hsp90 client proteins in human melanoma A-375 cells. Cancer Lett 318: 180-188, 2012.

13. Crissman HA and Steinkamp JA: Rapid, simultaneous measurement of DNA, protein, and cell volume in single cells from large mammalian cell populations. J Cell Biol 59: 766-771, 1973.

14. Rajan A, Kelly RJ, Trepel JB, et al: A phase I study of PF-04929113 (SNX-5422), an orally bioavailable heat shock protein 90 inhibitor, in patients with refractory solid tumor malignancies and lymphomas. Clin Cancer Res 17: 6831-6839, 2011.

15. Huang KH, Veal JM, Fadden RP, et al: Discovery of novel 2-aminobenzamide inhibitors of heat shock protein 90 as potent, selective and orally active antitumor agents. J Med Chem 52: 4288-4305, 2009.

16. Best OG, Che Y, Singh N, Forsyth C, Christopherson RI and Mulligan SP: The Hsp90 inhibitor SNX-7081 synergizes with and restores sensitivity to fludarabine in chronic lymphocytic leukemia cells with lesions in the TP53 pathway: a potential treatment strategy for fludarabine refractory disease. Leuk Lymphoma 53: 1367-1375, 2012.

17. Che Y, Best OG, Zhong L, et al: Hsp90 inhibitor SNX-7081 dysregulates proteins involved with DNA repair and replication and the cell cycle in human chronic lymphocytic leukemia (CLL) cells. J Proteome Res 12: 1710-1722, 2013.

18. Rice JW, Veal JM, Fadden RP, et al: Small molecule inhibitors of Hsp90 potently affect inflammatory disease pathways and exhibit activity in models of rheumatoid arthritis. Arthritis Rheum 58: 3765-3775, 2008.

19. Best OG, Singh N, Forsyth C and Mulligan SP: The novel Hsp-90 inhibitor SNX7081 is significantly more potent than 17-AAG against primary CLL cells and a range of haematological cell lines, irrespective of lesions in the TP53 pathway. Br J Haematol 151: $185-188,2010$.

20. Barta TE, Veal JM, Rice JW, et al: Discovery of benzamide tetrahydro-4H-carbazol-4-ones as novel small molecule inhibitors of Hsp90. Bioorg Med Chem Lett 18: 3517-3521, 2008.

21. Jin L, Xiao CL, Lu CH, et al: Transcriptomic and proteomic approach to studying SNX-2112-induced K562 cells apoptosis and anti-leukemia activity in K562-NOD/SCID mice. FEBS Lett 583: 1859-1866, 2009

22. Lee YH, Lee NH, Bhattarai G, et al: PPARgamma inhibits inflammatory reaction in oxidative stress induced human diploid fibloblast. Cell Biochem Funct 28: 490-496, 2010.

23. Bettger WJ, Boyce ST, Walthall BJ and Ham RG: Rapid clonal growth and serial passage of human diploid fibroblasts in a lipidenriched synthetic medium supplemented with epidermal growth factor, insulin, and dexamethasone. Proc Natl Acad Sci USA 78: 5588-5592, 1981.

24. Huang D, Gu Q, Ge H, et al: On the value of homology models for virtual screening: discovering hCXCR3 antagonists by pharmacophore-based and structure-based approaches. J Chem Inf Model 52: 1356-1366, 2012. 
25. Kawano T, Kobayakawa T, Fukuma Y, et al: A comprehensive study on the immunological reactivity of the Hsp90 molecular chaperone. J Biochem 136: 711-722, 2004.

26. Sreedhar AS, Kalmar E, Csermely P and Shen YF: Hsp90 isoforms: functions, expression and clinical importance. FEBS Lett 562: 11-15, 2004

27. Hong DS, Banerji U, Tavana B, George GC, Aaron J and Kurzrock R: Targeting the molecular chaperone heat shock protein 90 (HSP90): lessons learned and future directions. Cancer Treat Rev 39: 375-387, 2013.

28. Marzec M, Eletto D and Argon Y: GRP94: an HSP90-like protein specialized for protein folding and quality control in the endoplasmic reticulum. Biochim Biophys Acta 1823: 774-787, 2012.

29. Matassa DS, Amoroso MR, Maddalena F, Landriscina M and Esposito F: New insights into TRAP1 pathway. Am J Cancer Res 2: 235-248, 2012.

30. Kamal A, Thao L, Sensintaffar J, et al: A high-affinity conformation of Hsp90 confers tumour selectivity on Hsp90 inhibitors. Nature 425: 407-410, 2003.

31. Vilenchik M, Solit D, Basso A, et al: Targeting wide-range oncogenic transformation via $\mathrm{PU} 24 \mathrm{FCl}$, a specific inhibitor of tumor Hsp90. Chem Biol 11: 787-797, 2004.

32. Miyata Y: Hsp90 inhibitor geldanamycin and its derivatives as novel cancer chemotherapeutic agents. Curr Pharm Des 11: 1131-1138, 2005.
33. Harhaj EW and Sun SC: IKKgamma serves as a docking subunit of the IkappaB kinase (IKK) and mediates interaction of IKK with the human T-cell leukemia virus Tax protein. J Biol Chem 274: 22911-22914, 1999.

34. Mori N, Yamada Y, Ikeda S, et al: Bay 11-7082 inhibits transcription factor NF-kappaB and induces apoptosis of HTLVI-infected T-cell lines and primary adult T-cell leukemia cells. Blood 100: 1828-1834, 2002.

35. Zhou F, Zhang L, van Laar T, van Dam H and Ten Dijke P: GSK3 $\beta$ inactivation induces apoptosis of leukemia cells by repressing the function of c-Myb. Mol Biol Cell 22: 3533-3540, 2011.

36. Jin ZH, Kurosu T, Yamaguchi M, Arai A and Miura O: Hematopoietic cytokines enhance Chk1-dependent G2/M checkpoint activation by etoposide through the Akt/GSK3 pathway to inhibit apoptosis. Oncogene 24: 1973-1981, 2005.

37. Jia XZ, Yang SY, Zhou J, et al: Inhibition of CHK1 kinase by Go6976 converts 8-chloro-adenosine-induced G2/M arrest into $\mathrm{S}$ arrest in human myelocytic leukemia K562 cells. Biochem Pharmacol 77: 770-780, 2009.

38. Davis JM, Navolanic PM, Weinstein-Oppenheimer CR, et al: Raf-1 and Bcl-2 induce distinct and common pathways that contribute to breast cancer drug resistance. Clin Cancer Res 9: $1161-1170,2003$. 